\title{
Entre la ciencia social y la política pública: \\ reflexiones entorno a la \\ economía colombiana
}

FECHA DE RECEPCIÓN: 23 de agosto FECHA DE APROBACIÓN: 17 de octubre Pp. 86-101
Between Sacial Sciences and Public Policies: Reflections an the Colombian economy

Entre sciences saciales et politiques publiques: réflexions sur l'écanamie calombienne
IEntre a ciência sacial a a política pública: Reflexões em torno à economia colombiana
*Doctorado en Economía, Universidad Nacional Autónoma de México (UNAM). Maestro en Población y Desarrollo: Facultad Latinoamericana de Ciencias Sociales (FLACSO), Sede México. Experto en Gestión deProyectos y ONG: Universidad Complutense Madrid-España. Lic. en Economía: Universidad La Gran Colombia Bogotá- Colombia.

**Doctorando en Ciencias Empresariales, Universidad de Nebrija, España. MBA-Mágister en Administración, Universidad de La Salle. Especialista en Ingeniería de la ProducciónUniversidad Distrital Francisco fosé de Caldas. Ingeniero Mecánico, Universidad de América. 


\section{RESUMEN}

El vínculo entre las ciencias sociales y las políticas públicas, es uno de los aspectos de mayor interés entre quienes estudian el cómo traducir la evidencia empírica en decisiones de gobierno que beneficien determinada población. En este contexto, el lenguaje y los canales de comunicación entre investigadores y tomadores de decisiones, resultan claves para generar el acercamiento. En el presente artículo se reflexiona sobre este tema, tomando como ejemplo la relación entre ciencia y política pública en torno a la economía colombiana.

\section{ABSTRACT}

The connection between Social Sciences and Public Policies is one of the most interesting aspects for those who study how to transfer the empirical evidence of government decision making which benefits a certain population sector. In this context, language and communication channels between researchers and decision makers are key issues to generate a significant approach. In this research paper, we reflect on this given topic, taking into account the relationship between science and public policy, regarding the Colombian economy.

\section{RESUMÉÉ}

Cet article présente les évolutions de la CAN (Communauté Andine des Nations) en Le lien entre sciences sociales et politiques publiques est un des aspects les plus intéressants pour qui étudient les relations entre données empiriques et décisions gouvernementales bénéficiant une population déterminée. Dans ce contexte, le langage et les canaux de communication utilisés entre chercheurs et décideurs sont primordiaux pour créer un rapprochement. Cet article analysera cette thématique et prendra comme exemple la corrélation entre sciences sociales et politiques publiques relatives à l'économie colombienne.

\section{RESUMO}

A relação entre as ciências sociais e as políticas públicas é um dos aspectos de maior interesse entre aqueles que estudam o fato de como traduzir a evidência empírica em decisões de governo que beneficiem determinada população. Nesse contexto, a linguagem e os canais de comunicação entre pesquisadores e tomadores de decisões tornam-se chaves para gerar esta aproximação. Neste artigo reflete-se sobre este tema, tomando como exemplo a relação entre ciência e política pública em torno à economia colombiana.
Palabras claves

Comercio exterior

Políticas públicas

Ciencia y política

América Latina

Colombia

\section{Key words}

Foreign trade

Public policies

Science and politics

Latinamerica

Colombia

\section{Mots clefs}

Commerce Extérieur Politiques Publiques

Science Politique

Amérique Latine

Colombie

\section{Palavras-chave}

Comércio exterior

Políticas públicas

Ciência e política,

América Latina

Colômbia 


\section{INTRODUCción}

A diferencia de las ciencias exactas, que trabajan con datos que son previsibles en la mayoría de los casos, y cuyas hipótesis son factibles de verificar mediante experimentos de laboratorio, en los cuales se contrastan los resultados del caso de control frente a los mismos, en el de tratamiento. En las ciencias sociales, la comprobación de la hipótesis de investigación puede resultar más compleja y de menor sostenibilidad en el tiempo, dadas las características de la población con la cual se trabaja, es decir, seres humanos cuyos comportamientos pueden resultar en ocasiones previsibles mediante una lógica racional, sin embargo, en una buena parte de los casos, ser imprevisibles y manifestar cambios en un lapso muy corto de tiempo es de gran versatilidad.

Lo anterior indica, que en las ciencias sociales el nivel de caos o entropía asociado al sistema que se está estudiando, es mayor que el que puede presentarse cuando la población analizada o los datos con los que se trabaja, no varían significativamente en periodos muy cortos de tiempo. Esta situación impacta radicalmente en los resultados de la investigación, dado que para un buen conjunto de problemas de interés social, los hallazgos que se logran captar con los datos de hoy, pueden no ser válidos el día de mañana y por lo tanto, las hipótesis de investigación que se efectúan han de tener en cuenta el comportamiento cambiante de la sociedad.

El contexto relatado anteriormente, se complejiza cuando se intenta traducir los resultados de una investigación social en políticas públicas que beneficien determinada población y ayuden a resolver un problema específico, por varias razones: en primer lugar, la dificultad en la verificabilidad de los resultados de la investigación social y su sostenibilidad en el mediano plazo, hacen que estos sean incompatibles con las agendas públicas de los gobiernos, las cuales se rigen muchas veces por una lógica cortoplacista atada al interés electoral del tomador de decisiones. En segundo lugar, si las lógicas de acción de la ciencia social y la implementación de política pública son diferentes, la interacción que se logre entre ambas, manifestará divergencias en los lenguajes que se utilizan para transmitir, tanto los objetivos a perseguir, como los resultados a alcanzar. Esto lleva a plantear el tercer elemento, que complejiza la relación entre ciencia y política pública, el cual se refiere a la ausencia de puentes y canales de comunicación que permitan al investigador transmitir claramente los resultados de su investigación al político de turno.

En consecuencia, la traducción de los resultados de la investigación social en políticas públicas que beneficien determinada población y resuelvan un problema específico que se presenta, será el tema central de reflexión en el presente escrito. Con ello, el interrogante bajo el cual se girará la discusión es ¿Cómo lograr la transmisión de los resultados de la investigación social a los tomadores de decisiones, de tal forma que se traduzcan en políticas públicas que beneficien a la población? Para aterrizar el discurso, se citarán algunos resultados de investigaciones sobre comercio internacional en América Latina en la última década, con el fin de analizar de qué forma se han incorporado dichos resultados en la formulación e implementación de políticas públicas relacionadas con el sector externo.

Conforme a lo anterior, el presente artículo se desarrollará de la siguiente forma: en el primer apartado, se reflexionará en torno a las diferencias en las lógicas de acción y comprensión que existen entre las ciencias sociales y la operatividad de las políticas públicas, ello desde el punto de vista teórico con el énfasis en aspectos como las divergencias entre científicos sociales y tomadores de decisiones para transmitir sus objetivos y resultados y una aproximación al vínculo entre ciencia y política pública tomando como ejemplo el caso del comercio exterior.

En el segundo apartado, se explicará la aproximación metodológica para analizar el tema de discusión, indicando 
tanto las investigaciones que se utilizaron como fuente de análisis y que pueden contribuir al objetivo del presente escrito, como el supuesto que se busca corroborar con el análisis teórico empírico que se está realizando. En el tercer apartado, se desarrollará la idea que se busca argumentar haciendo la contrastación entre las investigaciones realizadas en relación con el tema y las políticas públicas que se han originado. El cuarto apartado, servirá como reflexión final sobre el interrogante que se planteó al inicio del escrito y que sirvió para formular el tema central del artículo.

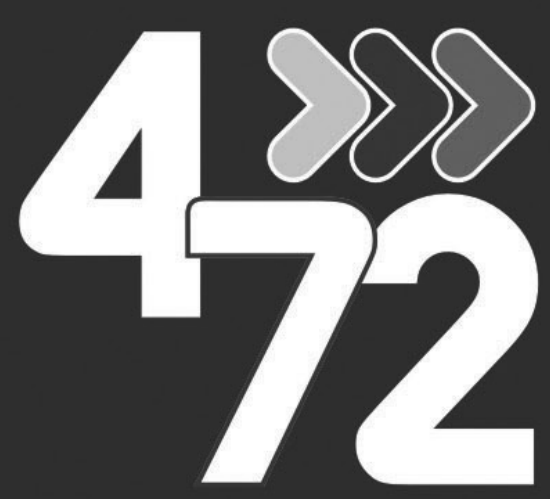

LARED POSTAL DE COLOMBIA

$w w w .4-72 . c 0 m . c 0$

\section{$\delta$ Línea de Atención al Cliente Nacional 018000111210}




\section{Ciencia social y política pública: LENGUAJES DIFERENTES, OBJETIVOS COMUNES}

$\mathrm{E}$ n la actualidad, las organizaciones multilaterales manifiestan una importante predisposición por impulsar el vínculo entre las ciencias sociales y la implementación de políticas públicas, la necesidad de establecer diálogos entre los tomadores de decisiones y los científicos sociales, se presenta como uno de los principales instrumentos en la construcción de consensos acerca del papel de la evidencia empírica en la investigación social y en la implementación de políticas (Milani, 2005). No obstante, el lenguaje y los intereses que persiguen tanto investigadores como políticos, difieren radicalmente con respecto a su dinámica de operación y el método que utilizan para el logro de sus objetivos. De esta forma, mientras que los científicos orientan regularmente su comportamiento a partir de una lógica deductiva o inductiva, buscando respuestas a los interrogantes que guiaron su investigación, los tomadores de decisiones, dado el contexto en el que se encuentran, operan en la mayoría de los casos bajo una lógica instrumental orientada a resolver su panorama electoral en el corto y mediano plazo.

Igualmente, mientras que el investigador valida sus resultados a partir del método científico y la contrastación de sus hipótesis, el político valida sus decisiones en función de la respuesta electoral que encuentra en sus electores.

Si tomamos estos dos extremos como parte de un mismo

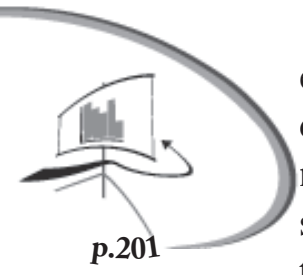
horizonte de estudio, encontramos en el medio una superestructura con diferentes niveles de análisis, la cual media las relaciones entre las ciencias sociales y la implementación de políticas públicas. Siguiendo a Milani (2005), estos niveles podrían desagregarse en las elecciones metodológicas/ontológicas del investigador, el proceso histórico de la institucionalización de las ciencias sociales, las motivaciones del tomador de decisiones y finalmente, las estructuras del gobierno que organizan el contexto en el cual se dan las relaciones entre los actores involucrados (Milani, 2005.p. 43). Cada uno de ellos, requiere de por sí una disertación completa con respecto a su papel en la relación ciencia-política, no obstante, el cuarto nivel podría orientar acerca de qué espacios se deben generar para que interactúen científicos sociales con tomadores de decisiones, con el fin de traducir los resultados de las investigaciones en políticas públicas que favorezcan a determinada población.

En efecto, el espacio en el cual se puede dar un encuentro entre los científicos sociales y los tomadores de decisiones se muestra permeado por el interés que tienen estos últimos con respecto a los resultados de las investigaciones para resolver un problema social identificado. Sin embargo, dado que en contadas ocasiones el político fundamenta sus decisiones en los hallazgos de la investigación social, y a cambio lo hace siguiendo el conflicto de intereses que ejercen los actores sociales, las empresas o las organizaciones de trabajadores en su agenda pública (O’Dwyer, 2004); la posibilidad de generar un encuentro entre los planificadores y ejecutores de políticas públicas y los científicos sociales, se reduce drásticamente.

A lo anterior, se añade el hecho que las relaciones entre ciencia y política se encuentran esquematizadas dentro de diferentes modelos que describen el interés, tanto de políticos como de investigadores. En algunos de ellos, la ciencia es utilizada como un instrumento de validación de las decisiones políticas, mientras que en otros, la relación descrita transciende hacia un diálogo entre los tomadores de decisiones y los científicos para resolver problemas sociales concretos (Weiss, 1986 citado en Milani, 2005).

De ser así, para acortar la distancia entre la investigación social y la implementación de política pública, han de ser solucionados cuando menos los siguientes aspectos: primero, homologación de los objetivos en las agendas tanto 
científicas como públicas, es decir, que tanto los programas de investigación social ${ }^{1}$ como los planes de gobierno persigan objetivos comunes orientados a la resolución de problemas sociales concretos. Segundo, compatibilizar los horizontes de tiempo que manejan, tanto la investigación como la política, dado que mientras la primera sigue lógicas de acción ejecutables en el mediano o largo plazo, la política pública se ejecuta regularmente en el corto tiempo. Tercero, el lenguaje de comunicación que utilizan tanto los científicos sociales como los políticos, debe ser homologado en códigos y terminologías que compartan y entiendan las dos partes de la relación que se está abordando, con el objetivo de traducir los resultados de las investigaciones en políticas públicas viables en el corto, mediano y largo plazo.

El discurso anterior, ha de ser plasmado en un caso concreto para que se perciba la lógica de lo que se está planteando. En este sentido, el tomar como ejemplo la reflexión en torno a las perspectivas económicas para el caso colombiano y su relación con

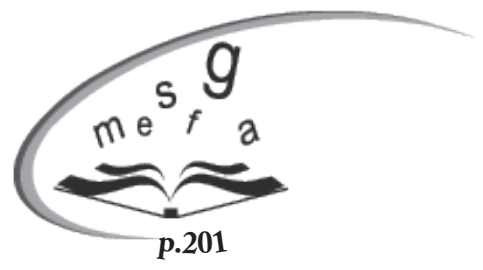
las políticas públicas implementadas, ayuda a entender los posibles obstáculos en la relación ciencia y política y la forma como podrían ser sobrepasados.

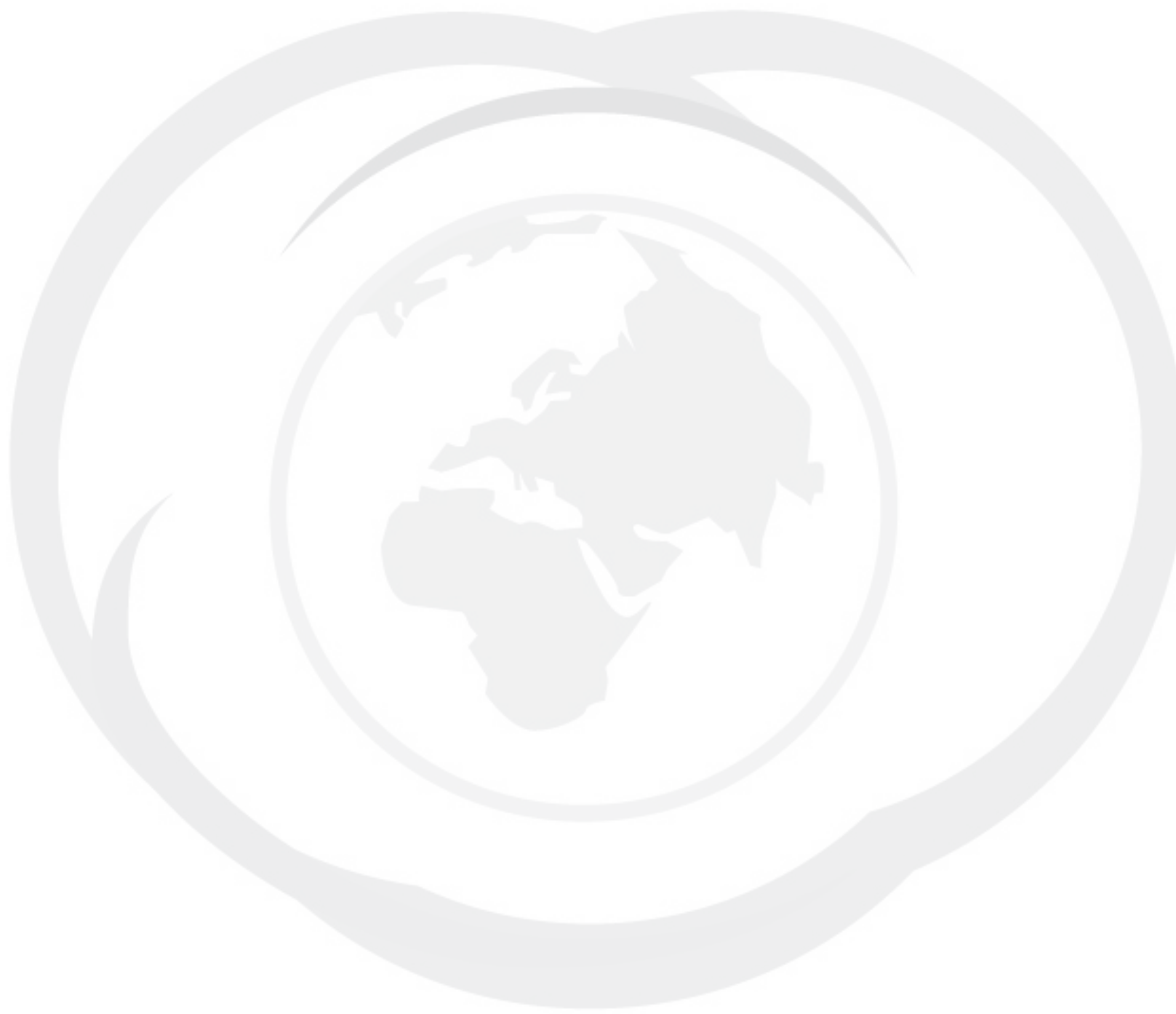

\footnotetext{
${ }^{1}$ Entendiendo el concepto de programa de investigación desde la perspectiva de Lakatos, en el cual las investigaciones y estudios que se realicen respecto de un tema particular contribuyen conjuntamente a construir el paradigma reinante (Lakatos, 1983).
} 


\section{REFLEXIÓN EN TORNO AL CASO DE LA ECONOMÍA COLOMBIANA: ¿QUÉ TANTO SE UTILIZA LA EVIDENCIA EMPÍRICA PARA FORMULAR POLÍTICA PÚBLICA?}

$\mathbf{E}$ $\mathrm{n}$ el presente apartado, se contrasta la reflexión en torno a las perspectivas de la economía colombiana en los últimos años con las políticas y programas de gobierno que se han planteado en materia de crecimiento económico. Esto permitirá por una parte, reflexionar sobre el distanciamiento que existe entre la evidencia empírica y la política pública para este caso en particular y por la otra, analizar los posibles canales de comunicación e intercambio de información entre los tomadores de decisiones y la academia que, o bien manifiestan serias debilidades, o no existen y deben construirse.

Hoy, las perspectivas económicas de América Latina son relativamente buenas, aún estando sujetas a incertidumbre y volatilidad en el contexto externo. Después de casi una década de expansión continuada, únicamente interrumpida durante 2009, las proyecciones más recientes indican que el Producto Interno Bruto (PIB) regional crecerá 3.2\% en 2012 y $4.0 \%$ en 2013 , lo que significa una desaceleración, al tiempo que se proyecta una caída de la inflación. En el corto plazo, este escenario supone un desempeño aceptable en comparación a la situación mundial y la continuidad de la estabilidad económica regional. Enfrenta también el riesgo de un impacto por la vía financiera en el caso que se reviertan las entradas de capitales, pero la región ha fortalecido sustancialmente su posición en términos de reservas internacionales que representan en promedio alrededor de un 16\% del PIB. (OCDE-CEPAL, 2013).

Colombia por su parte, enfrenta un escenario económico algo complejo debido al debilitamiento del crecimiento y a la actual incertidumbre de la economía mundial. Debido al menor dinamismo de la demanda externa y a la volatilidad en el precio de las materias primas de las cuales el país depende, las debilidades estructurales impedirían alcanzar un crecimiento económico mayor y más incluyente durante los próximos años. El fortalecimiento de las políticas macroeconómicas, los beneficios del auge de los productos básicos y las mejores condiciones de seguridad, han propiciado un vigoroso crecimiento económico desde principios de 2000.

Para mantener y consolidar los avances en la reducción de la pobreza y la desigualdad de los últimos años, se debe incrementar el nivel de crecimiento potencial y hacerlo más inclusivo. Algunos países latinoamericanos intentan combinar las tasas más altas de crecimiento económico de los últimos años, buscando la reducción en la pobreza y la desigualdad, a través de mejores políticas sociales y el incremento de los salarios, gracias a mercados laborales dinámicos. Es un buen intento, pero la región sigue siendo de las más desiguales del mundo y un 31\% de la población continúa siendo pobre.

En este contexto, las PyMEs son actores claves para incrementar el crecimiento potencial de América Latina. Representan alrededor del $99 \%$ del total de empresas y emplean cerca del $67 \%$ del total de trabajadores. No obstante, su contribución al PIB es relativamente baja, lo que revela deficiencias en los niveles de productividad de las mismas. Con la aplicación de políticas coherentes y coordinadas, las PyMEs podrían ser agentes del cambio estructural a través de su contribución al aumento de la productividad. (OCDE, 2013).

Un esfuerzo de esta naturaleza requiere de un cambio de enfoque en las políticas públicas hacia las PyMEs. Para que estas sean efectivas, se necesita una mayor coherencia, aticulación y coordinación entre las políticas de infraestructura, la provisión de servicios y las políticas sectoriales. En particular, las políticas en los ámbitos de financiamiento, competencias y formación, sistemas de innovación y difusión tecnológica y las políticas de articulación productiva, pueden ayudar a PyMEs a superar sus barreras. 
Las políticas públicas deben facilitar el desarrollo de las PyMEs y eliminar las barreras a las que se enfrentan, lo que permitiría cerrar las brechas con otras empresas con el objetivo de que actúen como agentes del cambio estructural. Para lograrlo, las políticas de PyMEs deben considerar tres factores relevantes: su aislamiento, que las deja imposibilitadas de añadir en su producción la escala y especialización necesarias. Por ello, a menudo las políticas son más efectivas cuando se enfocan sobre la cadena productiva en su conjunto del que las PyMEs forman parte.

En segundo lugar, se requiere de una coordinación significativa entre políticas sectoriales, así como políticas de

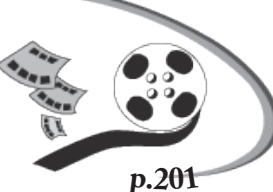
infraestructura y provisión de servicios para poder resolver los cuellos de botella relevantes para el desarrollo productivo de las PyMEs y su entorno.
En tercer lugar, combinada con esta mayor coordinación horizontal, tiene que ver con una mayor coordinación vertical, por el papel que juegan los actores locales y regionales en las políticas de apoyo a aglomerados y cadenas productivas a nivel subnacional. (OCDE-CEPAL,2013).

Según estadísticas del Banco de la República, Colombia en los últimos 10 años mostró un crecimiento económico que se refleja en el comportamiento de su PIB en el periodo 2004- 2007, el cual creció en promedio 5.8\%. Es decir, esta es una década en la que el país presentó grandes avances en materia económica, tanto así, que durante la crisis global de 2008 en la que se vieron afectadas algunas economías industrializadas, el país creció 1,6\% (tabla 1).

En promedio, el país presentó un crecimiento anual del PIB de 3,8\% y del PIB per cápita de 2,2\%. (Marrugo, 2013).

Tabla 1. PIB total y por habitantes, a precios constantes 2005, Colombia

\begin{tabular}{|c|c|c|c|c|c|c|}
\hline & \multicolumn{3}{|c|}{ Total } & \multicolumn{3}{c|}{ Por habitantes } \\
\hline Fin de & $\begin{array}{c}\text { Miles de } \\
\text { millones } \\
\text { de pesos }\end{array}$ & $\begin{array}{c}\text { Variación } \\
\text { anual \% }\end{array}$ & $\begin{array}{c}\text { Millones } \\
\text { de } \\
\text { dólares } \\
(\mathbf{2 0 0 5})\end{array}$ & Pesos & $\begin{array}{c}\text { Variación } \\
\text { anual \% }\end{array}$ & $\begin{array}{c}\text { Dólares } \\
\text { de 2005 }\end{array}$ \\
\hline 2000 & 284.761 & - & 122.701 & 7.006 .808 & - & 3.045 \\
\hline 2001 & 289.539 & 1,68 & 124.760 & 7.094 .190 & 0,39 & 3.057 \\
\hline 2002 & 296.789 & 2,50 & 127.884 & 7.181 .162 & 1,23 & 3.094 \\
\hline 2003 & 308.416 & 3,92 & 132.895 & 7.369 .789 & 2,63 & 3.176 \\
\hline 2004 & 324.866 & 5,33 & 139.982 & 7.667 .632 & 4,04 & 3.304 \\
\hline 2005 & 340.156 & 4,71 & 146.570 & 7.931 .153 & 3,44 & 3.417 \\
\hline 2006 & 362.938 & 6,70 & 156.387 & 8.361 .479 & 5,43 & 3.603 \\
\hline 2007 & 387.983 & 6,90 & 167.179 & 8.832 .464 & 5,63 & 3.806 \\
\hline $2008(p)$ & 401.744 & 3,55 & 173.108 & 9.037 .877 & 2,33 & 3.894 \\
\hline $2009(p)$ & 408.379 & 1,65 & 175.967 & 9.079 .360 & 0,46 & 3.912 \\
\hline $2010(p)$ & 424.719 & 4,00 & 183.008 & 9.332 .518 & 2,79 & 4.021 \\
\hline $2011(p)$ & 449.900 & 5,93 & 193.858 & 9.770 .961 & 4,70 & 4.210 \\
\hline
\end{tabular}

(p) provisional.

Nota: PIB en dólares de 2005= PIB en millones de pesos de 2005 sobre la tasa de cambio nominal promedio 2006.

Fuente. DANE-Dirección de síntesis y cuentas nacionales y Banco de la República. Estudios económicos-cuentas financieras. 
Analizando el comportamiento de los diferentes sectores de la economía, se puede inferir que la evolución obedece principalmente al fortalecimiento de la industria minera, la cual presenta variaciones positivas desde el año 2005, alcanzando mayor crecimiento durante 2008, 2009 y 2010 correspondientes al 9,6\%, 11,0\%, 12,2\%, respectivamente. (Marrugo, 2013).

El auge de los recursos naturales es positivo pero plantea desafíos económicos, sociales y ambientales. La minería, tiende a caracterizarse por requerir grandes capitales, no crear muchos puestos de trabajo y concentrarse regionalmente, por lo tanto, puede ampliar la brecha en la distribución de ingresos. La contaminación, también es un problema, especialmente en relación con las minas ilegales.

Las políticas estructurales son esenciales para aumentar la productividad y para ayudar a la economía en el juste del incremento en los términos de intercambio. La fuerte apreciación de la tasa de cambio vinculada al auge de los productos básicos, ha debilitado la competitividad de otros sectores transables. Para impulsar la productividad y evitar la adopción de nuevas medidas proteccionistas, habría que tomar como base una estrategia de tres ejes: la mejora del acceso a los mercados financieros, a través de una mejor regulación y una mayor competencia; la promoción de la inversión privada; y el fomento de una infraestructura de alta calidad, a través de un mejor marco institucional.

Los recientes tratados de libre comercio suscritos son un paso positivo, pero Colombia debería continuar reduciendo los aranceles de forma progresiva. Sin embargo, la economía colombiana se enfrenta a grandes desafíos a mediano plazo: impulsar el crecimiento de la productividad y reducir la desigualdad de los ingresos. Para hacer frente a esos desafíos, es necesario aplicar reformas estructurales. El auge de los recursos naturales, que probablemente perdurará varios años es positivo, pero también plantea desafíos en materia de política social, económica y ambiental. Ha impulsado la inversión extranjera, el crecimiento económico y los ingresos del gobierno, pero la mejora de los términos de intercambio y las correspondientes entradas de capital han contribuido a una fuerte apreciación de la tasa de cambio, lo que debilita la competitividad de otros sectores (OCDE, 2013).
Un mejor desempeño en el mercado laboral ayudará a reducir la desigualdad de los ingresos. La tasa de desempleo ha disminuido, pero aún sigue muy alta y la mayoría de personas que trabajan lo hacen en la economía informal y a menudo en ocupaciones de baja productividad. Los trabajajadores desempleados e informales, tienen pocas posibilidades de encontrar un empleo formal por regulaciones restrictivas del mercado de trabajo, en particular por unos costos laborales no salariales muy elevados y por un salario mínimo alto en comparación con la retribución media. (OCDE-CEPAL, 2013).

La inequidad en la distribución del ingreso se mide a través del Coeficiente de GINI (tabla 2). De acuerdo con las cifras, durante la década la tendencia de inequidad en Colombia alcanza niveles que van desde 0,56 en el año 2005 y 2010, hasta 0,61 en el 2002, pese al crecimiento de la economía.

Tabla 2. Coeficiente de GINI, 2000 - 2010, Colombia

\begin{tabular}{|c|c|c|}
\hline \multicolumn{3}{|c|}{ Coeficiente de GINI } \\
\hline \multicolumn{3}{|c|}{2000 - 2010 } \\
\hline Fin de & GINI & $\begin{array}{c}\text { Variación } \\
\text { anual \% }\end{array}$ \\
\hline 2000 & 0,59 & - \\
\hline 2001 & 0,58 & $-0,011$ \\
\hline 2002 & 0,61 & 0,046 \\
\hline 2003 & 0,58 & $-0,046$ \\
\hline 2004 & 0,58 & 0,007 \\
\hline 2005 & 0,56 & $-0,037$ \\
\hline 2006 & 0,59 & 0,045 \\
\hline 2007 & 0,59 & 0,004 \\
\hline 2008 & 0,57 & $-0,028$ \\
\hline 2009 & 0,57 & $-0,010$ \\
\hline 2010 & 0,56 & $-0,013$ \\
\hline
\end{tabular}

Fuente. Banco Mundial - Indicadores.

Esto evidencia que Colombia es un país desigual, tanto así, que la meta según el Departamento Nacional de Planeación (DNP) para el 2014, es llegar a tener un Coeficiente de GINI de 0,54 que, comparado con el promedio actual de América 
Latina, correspondiente a 0,53 , aún se sitúa por encima de este, razón por la cual el país se constituye como uno de los más desiguales del mundo al tener los ingresos concentrados en pocas manos (Rodríguez, 2011).
Al comparar la tasa de crecimiento del PIB en Colombia y el comportamiento de la tasa de crecimiento del coeficiente de GINI, se observa que entre el año 2000 y 2006, se presentó un crecimiento acelerado del PIB; sin embargo, el Coeficiente GINI alcanzó el máximo valor de la década en el año 2002 con 0,61 , lo que evidencia la fuerte inequidad que existe en el país y la poca realidad que refleja el PIB per cápita. (Marrugo, 2013).

Figura 1. PIB Crecimiento del PIB y comportamiento del coeficiente de GINI, Colombia

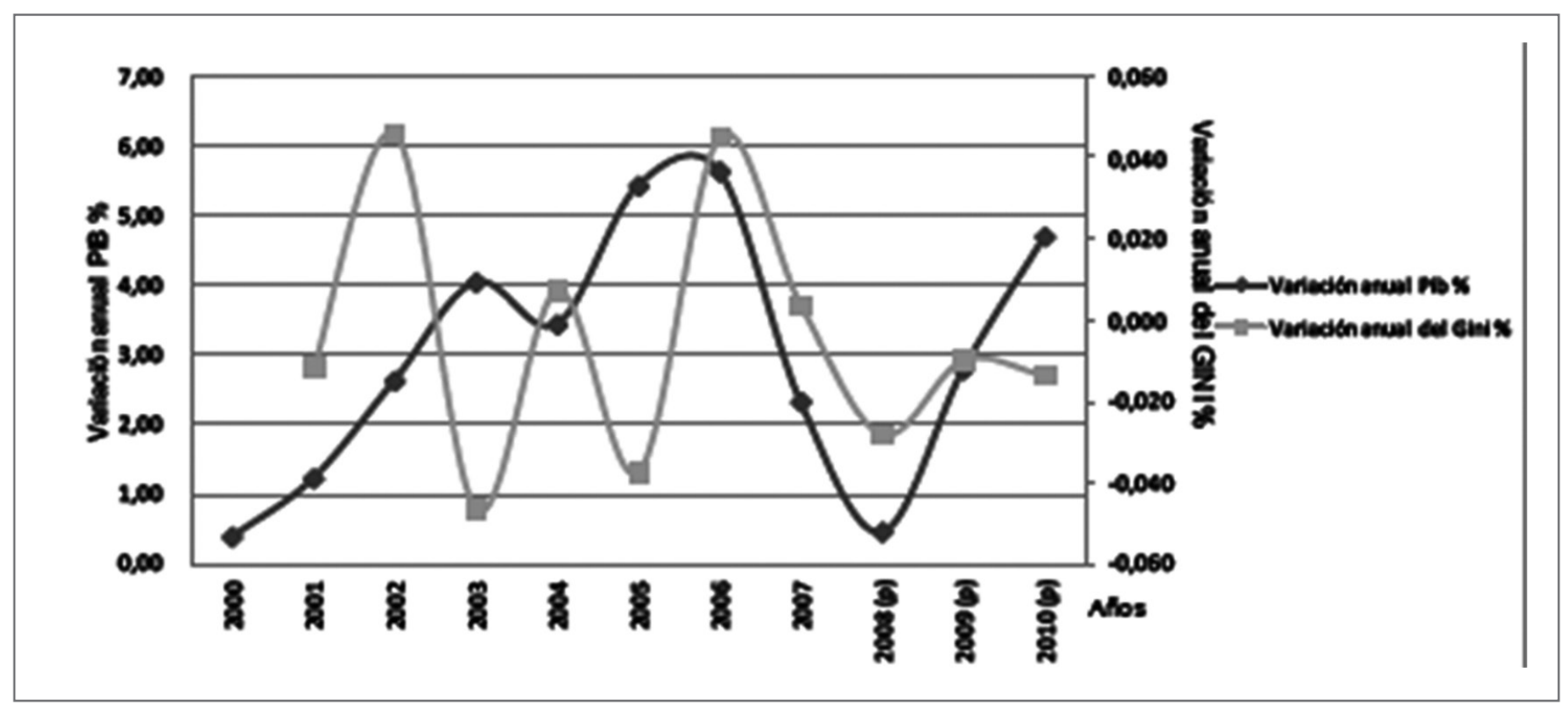

Fuente. PIB (Banco de la República), GINI (Banco Mundial).

La redistribución a través del sistema de impuestos y transferencias es muy baja. Se conseguiría reducir la desigualdad de los ingresos, aumentando el empleo formal mediante la disminución de los impuestos al trabajo y mediante la contención del aumento y la diferenciación del salario mínimo, fomentando un acceso equitativo a una educación de alta calidad para todos; disminuyendo los gastos fiscales que benefician principalmente a los ricos y reformando las transferencias a los hogares, que principalmente consisten en generosas pensiones a unos pocos ciudadanos relativamente acomodados (Marrugo, 2013). 
Figura 2. Evolución reciente de los precios de consumo y los salarios reales

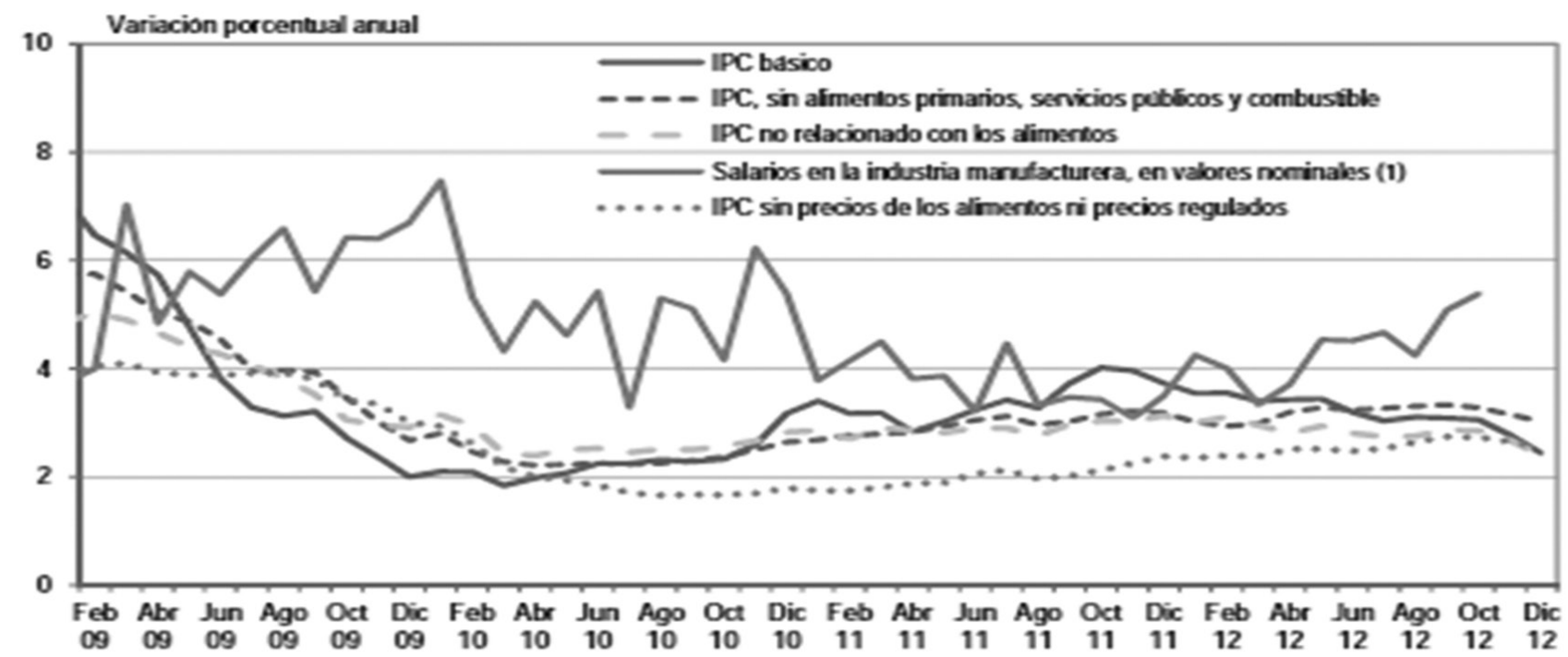

Fuente. Banco de la República.

A pesar del dinamismo que han tenido las exportaciones en los últimos años, la economía colombiana no tiene una apertura exportadora. La oferta exportable, está cada vez más concentrada en productos primarios y sus exportaciones no tradicionales se orientan a pocos mercados marcados por la inestabilidad. Adicionalmente, el notable dinamismo que se ha registrado recientemente en la atracción de inversión extranjera, se debilitó en 2009 dejando interrogantes sobre la sostenibilidad de esos flujos y su concentración en el sector de minas e hidrocarburos.

El rezago de la internacionalización de la economía colombiana, ha sido el resultado de la aplicación de políticas públicas que han restringido la productividad del país y han generado numerosos beneficios para sectores poco competitivos, como lo muestra el caso de la excesiva protección del sector agropecuario y la frustración generada por la aplicación del Programa Agro Ingreso Seguro. Casos así, ilustran cómo la preservación de los privilegios de unos pocos ha terminado perjudicando a toda la economía colombiana, minando su competitividad y sacrificando oportunidades de mayor crecimiento y empleo. (Reina, 2012). Factor que dejaría nuevamente la pregunta: ¿Dónde queda el nexo entre ciencia social y política pública en el caso de la economía colombiana?

El panorama relatado anteriormente, deja varios aspectos para reflexionar en torno a la relación entre ciencia social y política pública. Por una parte, si se analiza la asociación entre la evidencia empírica que se encontró y las políticas públicas que se han implementado, queda el interrogante de hasta qué punto las políticas y programas de desarrollo productivo que se han implementado para abatir la pobreza, fortalecer el tejido empresarial y aprovechar las ventajas del comercio exterior, han contado con un respaldo empírico que analice las condiciones socioeconómicas, laborales y productivas de las empresas involucradas, de tal suerte que las decisiones y apoyos específicos que se programen, sean pertinentes con respecto a la población beneficiaria y a su contexto particular.

Si se toman como referencia los estudios citados anteriormente y se comparan con las políticas económicas y productivas implementadas por las dos últimas administraciones en Colombia, se podría colegir que los tomadores de decisiones han estado ajenos a la situación de las empresas 
afectadas y sus características particulares, antes de diseñar programas viables y sostenibles. Es decir, que a nivel de pertinencia socioeconómica y laboral, los programas actuales manifiestan debilidades que los llevan a fallar en el mediano plazo y a no alcanzar sus propios objetivos.

Con respecto a la cobertura, encontramos igualmente un distanciamiento entre la evidencia empírica y las políticas públicas implementadas en el sector. En este sentido, la distancia entre estos dos factores puede estar muy correlacionada, tanto con el direccionamiento que se ha dado a los programas de apoyo productivo, como con la magnitud de la población afectada y la incidencia de factores externos que afectan el problema a resolver.

Ahora bien, respecto a los canales de comunicación que pueden existir entre los tomadores de decisiones, la sociedad y los investigadores sociales en Colombia, es pertinente aclarar que estos dependen del contexto particular en el cual se circunscribe cada investigación y de los espacios de intercambio que se abran para discutir un tema específico. La experiencia que cada investigador o tomador de decisiones pueda relatar acerca de la relación entre ciencia social y política pública, dado que es una vivencia personal que se relaciona con un contexto y momento histórico específico, puede llegar a manifestar un alto grado de subjetividad atada a la percepción que tenga el individuo con respecto a los vínculos que estableció con la contraparte.

Las divergencias en la perspectiva de análisis utilizada, la inconveniencia política de las conclusiones del estudio, así como la carencia de sólidos nexos generados a partir de redes sociopolíticas, pueden estar afectando la difusión y aplicación de los resultados de la investigación. Con ello, la pregunta planteada al inicio del escrito da lugar a un nuevo interrogante: de existir la forma para difundir y traducir los hallazgos empíricos en políticas públicas concretas, ¿cómo lograr que estos coincidan con los intereses políticos del gobierno de turno y sean incluidos en su agenda de mediano o largo plazo? 


\section{CONCLUSIÓN}

E $\mathrm{n}$ el primer apartado del presente escrito, se reflexionó en torno a la relación entre las ciencias sociales y las políticas públicas, tomando como punto de partida las divergencias que existen con respecto a la lógica de acción, lenguaje y metodología que utilizan tanto los tomadores de decisiones, como los investigadores sociales para transmitir sus objetivos y resultados. En este sentido, se detectó que existen diferentes niveles de análisis en esta relación, cada uno de los cuales ha de ser estudiado con detalle, verificando los distintos aspectos que pueden potenciar o desestimular un acercamiento entre ciencia y política. Así mismo, se abordó la relación ciencia social y política pública, tomando como ejemplo el caso de las perspectivas económicas para Colombia; allí, se indicó que si bien la evidencia empírica invita a plantear un conjunto de recomendaciones en materia de políticas públicas para cada sector, al momento de ser implementadas pueden encontrar tanto beneficiarios como fuertes detractores, dejando en el medio al tomador de decisiones quien regularmente optará por la decisión que maximice tanto su beneficio electoral como la utilidad socioeconómica al menor costo posible.

Luego, en el análisis de perspectiva económica para Colombia, se observó que de acuerdo con los estudios realizados sobre la situación de la economía colombiana, existe una distancia significativa entre la evidencia de los datos empíricos recabados y las políticas públicas y programas de gobierno que se han implementado en materia de desarrollo productivo.

Siguiendo lo anterior, se indicó que el acceso y utilización de canales de comunicación entre investigadores y tomadores de decisiones como instrumento en la traducción de los resultados de la investigación en políticas concretas, se encuentra afectado por el contexto en el cual esté inmerso cada investigador y el tema particular de su estudio. El análisis muestra que la aplicación de los resultados de la investigación social en políticas públicas que beneficien a una población determinada, puede ser la utopía a alcanzar en el largo plazo para algunos investigadores, mientras que para el presente queda la satisfacción de haber cumplido con la responsabilidad social de exponer parte de la realidad y haber planteado propuestas con fundamento para cambiarla. Con ello, el vínculo entre las ciencias sociales y las políticas públicas, habrá de reforzarse mediante diversos aspectos:

- Construcción de espacios abiertos de interacción entre los tomadores de decisiones y los científicos sociales para debatir los temas de interés social.

- Ampliación de los canales de difusión de los resultados de las investigaciones sociales (favorables y desfavorables a los intereses del gobierno).

- Traducción de los resultados de la investigación social en mecanismos e instrumentos de políticas públicas favorables a la población.

- Hablar un mismo lenguaje entre investigadores y tomadores de decisiones, con el fin de homogenizar tanto los objetivos como los resultados que persiguen ambas partes. 


\section{REFERENCIAS}

Bobrow, D.B y J.S. Dryzek, (1987) Policy Analysis by Design. Pittsburgh UPP.

De Soto, H. (2000) El Misterio del Capital. ED. Norma, Lima Perú

Marrugo, V. (2013). Crecimiento económico y desarrollo humano en Colombia (2000-2010). Revista de economía del Caribe N. 11

Gamarra, E. (2004) Evaluación de la vulnerabilidad de conflicto en Bolivia. Latin American and Caribbean Center Florida Internacional University. USAID. Florida 2004.

Hart, K. (1973) Informal income opportunities and urban employment in Ghana, en Journal of Modern African Studies, N. 11, 1973.

Hernández, G. (2004) 25 años de empleo, ocupación e informalidad en México. Ponencia presentada en el seminario sobre Pobreza Moderada e Informalidad, FLACSO - IBERGOB, octubre de 2004

Lakatos, I. (1983) La metodología de los programas de investigación cientifica. Ed. Alianza Universidad, Madrid, España

Milani, C. (2005) Evidence based policy research: critical review of some international programs on relationship between social science research and policy making, en Policy papers. Ed. MOST - UNESCO, Paris - Francia.

O’Dwer, L. (2004) A critical review of evidence based policy making. Australian Housing and Urban Research Institute, AHURI, Final Report, N. 58.

OCDE (2013) Estudios económicos de la OCDE Colombia. Evaluación económica y visión general.

OCDE-CEPAL (2013) Perspectivas económicas de América Latina 2013. Políticas de pymes para el cambio estructural.

Olson, M. (2000) Power and Prosperity: Outgrowing Communist and Capitalism Dictatorships. Ed. Basic Books New York 2000

OMPE (2007) Resultados del censo de actividades económicas del municipio de La Paz 2007. Oficialía Mayor de Promoción Económica del gobierno municipal de la ciudad de La Paz, La Paz Bolivia 2007.

Parra, L. (2009a) Empresarialidad Informal y contexto regional: Bolivia en los años 2000 y 2005. The Latinamericanist review, Issue March 2009, Ed. Blackwell Publishing - SECOLAS Annals, Pittsburg University Press.

Parra, L. (2009b) El papel del estado en la promoción de la empresarialidad informal: reflexiones entorno al caso de Bolivia. Ponencia presentada en el XXVIII congreso de Latin American Studies Association (LASA) realizado en Río de Janeiro, Brasil en Junio de 2009. 
Portes, A. (1995) En torno a la Informalidad: Ensayos sobre teoría y medición de la economía no regulada. FLACSO México 1995.

Reina, M. (2012) Internacionalización de la economía colombiana: comercio e inversión. Fedesarrollo, Corporación Andina de Fomento- CAF.

, y Haller, W. (2004) La economía informal, en Políticas Sociales N. 100, Ed. CEPAL, Santiago de Chile.

Tokman, V. (1991) El Enfoque PREALC, en Tokman, V. El Sector Informal en América Latina, dos décadas de análisis, Consejo nacional para la cultura y las artes, México, 1991.

(2001) Políticas de Empleo en la Nueva Era Económica. Trabajo presentado en el Seminario "La teoría del desarrollo en los albores del siglo XXI", organizado por La CEPAL en Santiago, Agosto del 2001.

Weiss, C. (1986) The many meanings of research utilization, in Bulme, M Ed. Social Science and Policy. London. 


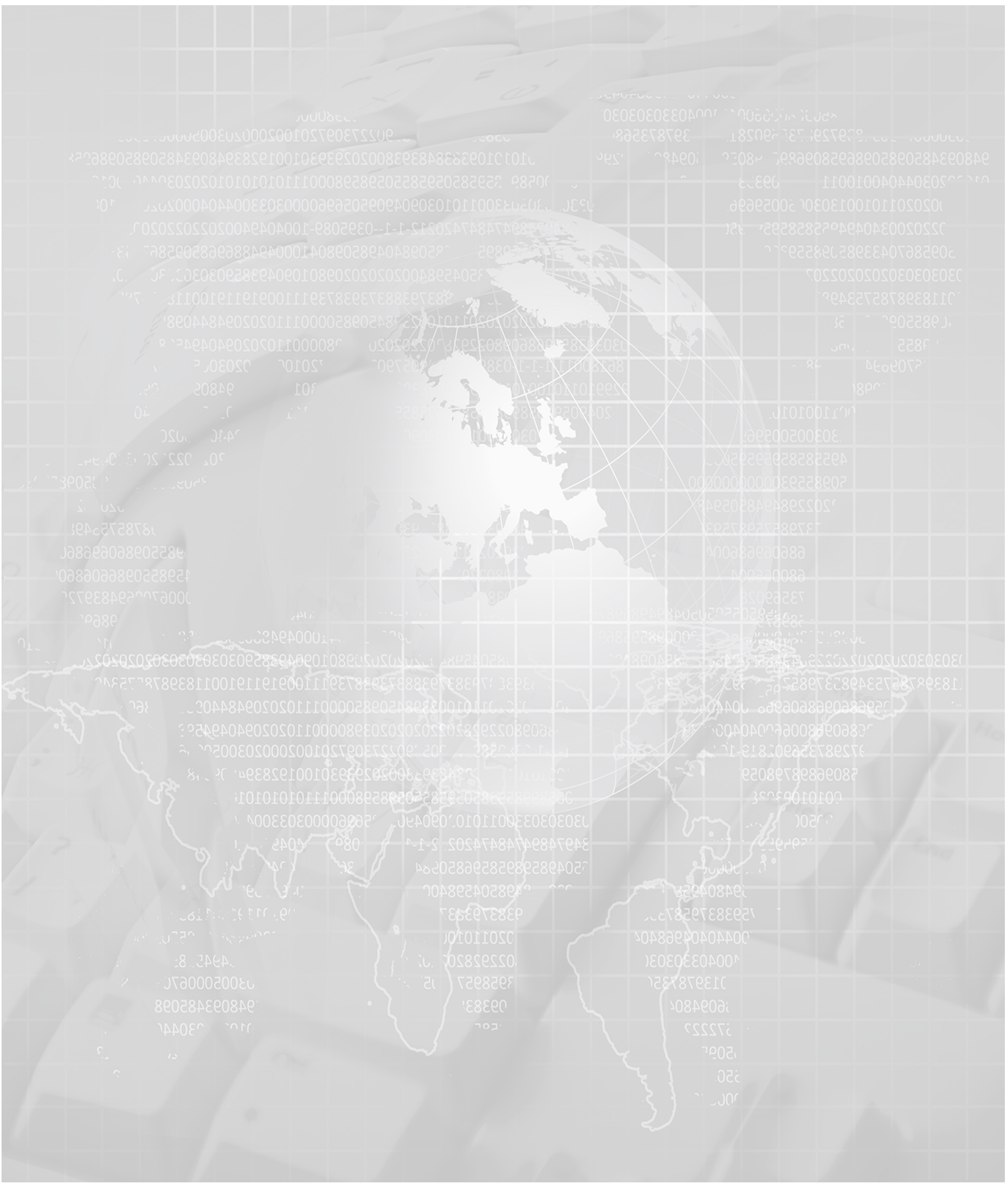

